\title{
Gender difference in cycling speed and age of winning performers in ultra-cycling - the 508-mile "Furnace Creek" from 1983 to 2012
}

\author{
Rüst, Christoph Alexander ; Rosemann, Thomas ; Lepers, Romuald ; Knechtle, Beat
}

\begin{abstract}
We analysed (i) the gender difference in cycling speed and (ii) the age of winning performers in the 508-mile "Furnace Creek 508". Changes in cycling speeds and gender differences from 1983 to 2012 were analysed using linear, non-linear and hierarchical multi-level regression analyses for the annual three fastest women and men. Cycling speed increased non-linearly in men from $14.6(\mathrm{~s}=0.3) \mathrm{km} \cdot \mathrm{h}(-1)(1983)$ to $27.1(\mathrm{~s}=0.7) \mathrm{km} \cdot \mathrm{h}(-1)$ (2012) and non-linearly in women from $11.0(\mathrm{~s}=0.3) \mathrm{km} \cdot \mathrm{h}(-1)(1984)$ to $24.2(\mathrm{~s}=0.2) \mathrm{km} \cdot \mathrm{h}(-1)(2012)$. The gender difference in cycling speed decreased linearly from $26.2(\mathrm{~s}=0.5) \%(1984)$ to $10.7(\mathrm{~s}=1.9) \%(2012)$. The age of winning performers increased from $26(\mathrm{~s}=2)$ years $(1984)$ to $43(\mathrm{~s}=11)$ years $(2012)$ in women and from 33 $(s=6)$ years (1983) to $50(s=5)$ years $(2012)$ in men. To summarise, these results suggest that (i) women will be able to narrow the gender gap in cycling speed in the near future in an ultra-endurance cycling race such as the "Furnace Creek 508" due to the linear decrease in gender difference and (ii) the maturity of these athletes has changed during the last three decades where winning performers become older and faster across years.
\end{abstract}

DOI: https://doi.org/10.1080/02640414.2014.934705

Posted at the Zurich Open Repository and Archive, University of Zurich

ZORA URL: https://doi.org/10.5167/uzh-97173

Journal Article

Accepted Version

Originally published at:

Rüst, Christoph Alexander; Rosemann, Thomas; Lepers, Romuald; Knechtle, Beat (2015). Gender difference in cycling speed and age of winning performers in ultra-cycling - the 508-mile "Furnace Creek" from 1983 to 2012. Journal of Sports Sciences, 33(2):198-210.

DOI: https://doi.org/10.1080/02640414.2014.934705 


\section{Gender difference in ultra-cycling}

\section{Gender difference in cycling speed and age of winning performers in ultra-cycling - the 508-mile 'Furnace Creek' from 1983 to 2012}

Christoph Alexander Rüst ${ }^{1}$, Thomas Rosemann ${ }^{1}$, Romuald Lepers ${ }^{2}$, Beat Knechtle ${ }^{3}$ Institute of General Practice and for Health Services Research, University of Zurich, Zurich, Switzerland

2 INSERM U1093, Faculty of Sport Sciences, University of Burgundy, Dijon, France 3 Gesundheitszentrum St. Gallen, St. Gallen, Switzerland 


\begin{abstract}
We analysed (i) the gender difference in cycling speed and (ii) the age of winning performers in the 508-mile 'Furnace Creek 508'. Changes in cycling speeds and gender differences from 1983 to 2012 were analysed using linear, non-linear and hierarchical multi-level regression analyses for the annual three fastest women and men. Cycling speed increased non-linearly in men from $14.6(s=0.3) \mathrm{km} \cdot \mathrm{h}^{-1}(1983)$ to $27.1(s=0.7) \mathrm{km} \cdot \mathrm{h}^{-1}(2012)$ and non-linearly in women from $11.0(s=0.3) \mathrm{km} \cdot \mathrm{h}^{-1}(1984)$ to $24.2(s=0.2) \mathrm{km} \cdot \mathrm{h}^{-1}$ (2012). The gender difference in cycling speed decreased linearly from $26.2(s=0.5) \%(1984)$ to $10.7(s=1.9) \%$ (2012). The age of winning performers increased from $26(s=2)$ years $(1984)$ to $43(s=11)$ years $(2012)$ in women and from $33(s=6)$ years $(1983)$ to $50(s=5)$ years $(2012)$ in men. To summarize, these results suggest that $(i)$ women will be able to narrow the gender gap in cycling speed in the near future in an ultra-endurance cycling race such as the 'Furnace Creek 508' due to the linear decrease in gender difference and (ii) the maturity of these athletes has changed during the last three decades where winning performers become older and faster across years.
\end{abstract}

Key words: ultra-endurance, woman, man 


\section{Introduction}

Ultra-endurance races are mainly held in swimming (Eichenberger, et al., 2012a, 2012d, 2013; Fischer, Knechtle, Rüst, \& Rosemann, 2013), cycling (Knechtle, Enggist, \& Jehle, 2005; Knechtle, Wirth, Knechtle, Rüst, Rosemann, \& Lepers, 2012b; Rüst, Knechtle, Rosemann, \& Lepers, 2013b; Zingg, Knechtle, Rüst, Rosemann, \& Lepers, 2013a), running (Eichenberger, Knechtle, Rüst, Rosemann, \& Lepers, 2012b; Hoffman, 2010; Hoffman \& Wegelin, 2009), and triathlon (Herbst, et al., 2011; Lepers, 2008; Lepers, \& Maffiuletti, 2011; Rüst, Knechtle, Knechtle, Rosemann, \& Lepers, 2012b).

Over the last decades, the gender difference in ultra-endurance performance - defined as any endurance performance lasting for six hours or longer (Zaryski, \& Smith, 2005) - was of particular interest in ultra-running (Bam, Noakes, Juritz, Dennis, 1997; Cheuvront, Carter, Deruisseau, \& Moffatt, 2005; Coast, Blevins, \& Wilson, 2004; Hoffman, 2008). Several studies addressed the question whether the gender difference in running performance would decrease with increasing race distance (Bam, Noakes, Juritz, \& Dennis, 1997; Cheuvront, Carter, Deruisseau, \& Moffatt, 2005; Coast, Blevins, \& Wilson, 2004; Hoffman, 2008; Speechly, Taylor, \& Rogers, 1996). Some data suggested that women matched with men for running performance in $42.2 \mathrm{~km}$ or $56 \mathrm{~km}$ distances were able to run faster than men in longer distances such as 96km (Bam, Noakes, Juritz, \& Dennis, 1997; Speechly, Taylor, \& Rogers, 1996).

The gender difference in ultra-endurance performance seemed to be influenced by the duration (Knechtle, Knechtle, \& Lepers, 2011a; Rüst, Knechtle, Knechtle, Rosemann, \& 
Lepers, 2012d; Rüst, Knechtle, Rosemann, \& Lepers, 2013b) and the locomotion mode (Lepers, 2008; Lepers, \& Maffiuletti, 2011) of the performance. Coast, Blevins, and Wilson (2004) compared the world's best running performances from $100 \mathrm{~m}$ to $200 \mathrm{~km}$. Men were $\sim 12.4 \%$ faster than women and longer running distances were associated with greater gender differences. In ultra-triathlons, the world's best men were $\sim 19 \%$ faster than the world's best women in Double Iron and Triple Iron, and $~ 30 \%$ faster in Deca Iron ultra-triathlon (Knechtle, Knechtle, \& Lepers, 2011a). With increasing length of an ultra-triathlon distance, the world's best women became slower compared to the world's best men.

The changes in gender differences in performances across time in ultra-endurance events have been examined for different disciplines. There seemed to be differences in the gender difference in performance depending upon the locomotion mode. In ultra-distance swimming, women might achieve similar performances compared to men in both indoor pool swimming (Eichenberger, et al., 2012c) and open-water swimming (Eichenberger, et al., 2012a). Indeed, in the $35 \mathrm{~km}$ 'Manhattan Island Marathon Swim', the ten fastest women were faster than the ten fastest men (Knechtle, Rosemann, Lepers, \& Rüst, 2014). In running, race times of women improved relative to men in $161 \mathrm{~km}$ ultra-marathons held through the $1980 \mathrm{~s}$ in North America (Hoffman, 2010). Over the past two decades, race times were stable with the fastest women running $\sim 20 \%$ slower than the fastest men (Hoffman, 2010). Similar results were reported for the $78 \mathrm{~km}$ 'Swiss Alpine Marathon', where the gender difference in performance decreased from $22 \%$ (1998) to $17 \%$ (2011) (Eichenberger, Knechtle, Rüst, Rosemann, \& Lepers, 2012b). Obviously, women seemed to be able to close the gender gap for certain disciplines such as ultra-swimming and in shorter ultra-running distances. 
For cycling, however, little is known regarding the gender difference in performance (Pozzi, Knechtle, Knechtle, Rosemann, Lepers, \& Rüst, 2014; Schumacher, Mueller, \& Keul, 2001; Rüst, Knechtle, Rosemann, \& Lepers, 2013b; Zingg, Knechtle, Rüst, Rosemann, \& Lepers, 2013a). Schumacher, Mueller and Keul (2001) studied race results of the World Track Cycling Championships in 200m, 1,000m, individual and team pursuit races for elite and junior athletes and reported a gender difference of $11(s=1.8) \%$ for all disciplines and ages. Considering ultra-cycling, the gender difference in performance has mainly been investigated in the $180 \mathrm{~km}$ cycling split in long-distance triathlons such as 'Ironman Hawaii' (Lepers, 2008; Lepers, \& Maffiuletti, 2011). The gender difference in the $180 \mathrm{~km}$ cycling performance in 'Ironman Hawaii' ranged between $12.7(s=2.0) \%$ (Lepers, 2008) and $15.4(s=0.7) \%$ (Lepers, \& Maffiuletti, 2011). Drafting (i.e. cycling in a close group in order to reduce the overall effect of drag) is forbidden in 'Ironman Hawaii'. However, women seemed not to profit from drafting. In a 24-h cycling draft-legal event, the sex difference for the fastest finishers was $20 \%$ (Pozzi, Knechtle, Knechtle, Rosemann, Lepers, \& Rüst, 2014). The present study intends to investigate the gender difference in cycling speed of winning performers in a non-drafting ultra-cycling race, the 508-mile 'Furnace Creek 508'. The 'Furnace Creek 508' is named after the total length of its course of 508miles (818km) and the location of its midpoint near Furnace Creek in California. The 'Furnace Creek 508' is an ultra-cycling race taking place since 1983 in Southern California, USA. This race was once a qualifying event for the 'Race across America' (RAAM), the longest non-stop ultra-cycling race in the world.

Apart from the gender difference, the age of peak endurance performance is of interest for athletes to plan a career (Eichenberger, Knechtle, Rüst, Rosemann, \& Lepers, 2012b; Hunter, Stevens, Magennis, Skelton, \& Fauth, 2011; Rüst, Knechtle, Knechtle, Rosemann, \& Lepers, 
2012b; Schulz, \& Curnow, 1988). In marathon running, the age of peak performance was $\sim 30$ years for both women and men (Hunter, Stevens, Magennis, Skelton, \& Fauth, 2011). Ultraendurance performances, however, are achieved at higher ages. Eichenberger, Knechtle, Rüst, Rosemann, and Lepers (2012b) found at the 78km 'Swiss Alpine Marathon' an unchanged age of peak running performance of $38(s=5)$ years for the top ten men but an increase from $32(s=5)$ years to $39(s=6)$ years in women. Male top ten Ironman triathletes competing in 'Ironman Switzerland' with $33(s=3)$ years were of the same age like women with $34(s=4)$ years (Rüst, Knechtle, Knechtle, Rosemann, \& Lepers, 2012c). Across years, however, the age of peak Ironman performance remained unchanged $31(s=3)$ years for the annual top ten men but increased from $30(s=4)$ to $36(s=5)$ years in women. In ultra-triathlon, Knechtle, Rüst, Knechtle, Rosemann and Lepers (2012b) showed no change for the fastest men in Triple Iron ultra-triathlon and Deca Iron ultra-triathlon between 1992 and 2010. The age of peak performance was $35.8(s=4.5)$ years in Triple Iron and $38.0(s=6.8)$ years in Deca Iron ultratriathlon.

To date, no study investigated the gender difference in cycling speed and the age of winning performers in a non-drafting ultra-cycling race. In particular, no study investigated these changes across time. The first aim of the present study was to analyse the changes in cycling speed in the 'Furnace Creek 508' from 1983-2012. The second aim was to identify the age of winning performers. Based upon recent reports that the gender difference was higher in ultramarathon races (Knechtle, Knechtle, \& Lepers, 2011a; Rüst, Knechtle, Knechtle, Rosemann, \& Lepers, 2012b) compared to marathons, we firstly hypothesized that men would be considerably faster in ultra-cycling than existing reports on cycling split performances in triathlon (Lepers, 2008; Lepers, \& Maffiuletti, 2011) and duathlon (Rüst, et al., 2013a). 
Secondly, we hypothesized that the age of peak ultra-cycling performance would be at a higher age than reported for marathoners. 


\section{Materials and methods}

\section{Ethics and data sampling}

The present study was approved by the Institutional Review Board of St. Gallen, Switzerland, with a waiver of the requirement for informed consent given that the study involved the analysis of publicly available data. The data set was obtained from the race website www.the508.com of 'Furnace Creek 508'. All race results were complete from 1983 to 2012. In 2013, the race was not held over the full distance.

\section{The race}

The 'Furnace Creek 508' is an ultra-marathon cycling race held annually in Southern California, USA, since 1983. The race was founded by John Marino in 1983 and known as 'The Toughest 48 hours in Sport' and 'The Great American Bike Race'. It is the world's premier ultra-cycling race. The 'Furnace Creek 508' is named after the total length of its course of 508 miles $(818 \mathrm{~km})$ and the location of its midpoint (i.e. near Furnace Creek, California). The race was once a qualifying event for the 'Race Across AMerica' (RAAM), but has not been since 2003 due to rule changes. From 1983 to 1984, the race was called the 'John Marino Open', from 1985 to 1986 the 'John Marino Open West', from 1987 to 1991 the 'RAAM Open West' and since 1992 'Furnace Creek 508'. The course has a total elevation gain of over 36,000 feet $(11,000 \mathrm{~m})$ and crosses ten mountain passes. Its route starts in Santa Clarita, California (i.e. 25miles north of Los Angeles), goes northeast to Towne Pass and drops into Death Valley, traverses Death Valley in the southern direction, crosses Mojave Desert and ends at Joshua Tree National Park, Twentynine Palms, California. In order to be declared an official finisher, an entrant must cross the finish line within $48 \mathrm{~h}$. 


\section{Data analysis}

Race times and ages of all cyclists who ever participated in the 'Furnace Creek' between 1983 and 2012 were analysed. Data were available from 836 athletes, including 750 men and 86 women who had successfully finished. Race times were converted to cycling speed $\left(\mathrm{km} \cdot \mathrm{h}^{-1}\right)$ prior to analysis by calculating [race distance] / [race time]. To examine the changes over time in cycling speed, the sex difference in cycling speed and the age of peak cycling speed, the cycling speed and the age of the annual female and male winners and the annual top three women and men were determined. The gender difference was calculated using the equation ([value (cycling speed or age) in women] - [value (cycling speed or age) in men]) / [value (cycling speed or age) in men] $\times 100$, where the gender difference was calculated for every pair of equally placed athletes. For calendar years with less than the investigated number of female and male finishers, the respective group was excluded and the calculation of gender difference was waived. These were the years 1983, 1985, 1988-1997, 2000, 2004, and 2009. Additionally, the performance of the overall first ever, the overall top three ever and the overall top ten women and men ever were analysed.

\section{Statistical analysis}

Each set of data was tested for normal distribution and for homogeneity of variances prior to statistical analyses. Normal distribution was tested using a D'Agostino and Pearson omnibus normality test and homogeneity of variances was tested using a Levene's test. Trends in participation were analysed using regression analyses with linear and exponential growth equation models. For each set of data (e.g. each age group), both models were compared using Akaike's Information Criteria (AICc) to determine the model with the highest probability of correctness. Single and multi-level regression analyses investigated changes in cycling speed, in sex difference in cycling speed and in the age of the winning performers. A 
hierarchical regression model avoided the impact of a cluster-effect on results where a particular athlete finished the race more than once. Regression analyses for cycling speed and sex difference in cycling speed were corrected for age of the athletes to prevent a misinterpretation of the 'age-effect' as a 'time-effect' since age is an important predictor variable in ultra-endurance performance such as 100km ultra-marathon running (Knechtle, Knechtle, Rosemann, \& Lepers, 2010b). The regression models for cycling speed and sex difference in cycling speed were also corrected with environmental temperatures (i.e. the lowest, the median and the highest air temperature during every race) since environmental conditions such as extreme heat may impair endurance (Abbiss, et al., 2010; Pfeiffer, \& Abbiss, 2011) and ultra-endurance performances (Parise, \& Hoffman, 2011; Wegelin, \& Hoffman, 2011). Historical weather data with ambient air temperatures for the daily lowest, the median and the daily highest temperatures during the race were retrieved from www.almanac.com/weather/history (Table 1). Since the changes in gender differences in endurance performance are assumed to be non-linear (Reinboud, 2004), we additionally calculated the non-linear regression model. When the best-fit model was non-linear (i.e. polynomial) regression, we compared the non-linear to the linear model using AIC and F-test in order to show which model would be the most appropriate to explain the trend of the data. To find significant differences between two groups a Student's $t$-test with Welch's correction was used in case of significant different variances between the groups. Statistical analyses were performed using IBM SPSS Statistics (Version 19, IBM SPSS, Chicago, IL, USA) and GraphPad Prism (Version 5, GraphPad Software, La Jolla, CA, USA). Significance was accepted at $P<0.05$ (two-sided for $t$-tests). Data in the text are given as mean \pm standard deviation $(s)$. 


\section{Results}

A total of 1,321 cyclists started in the race including 1,172 men (88.8\%) and 149 women $(11.2 \%)$. Of the starters, $836(62.2 \%)$ cyclists finished successfully the race within the time limit. Among the finishers, $750(89.9 \%)$ were men and $86(10.1 \%)$ were women. The number of female and male finishers increased exponentially (Figure 1).

\section{Changes in cycling speeds across years}

For the annual fastest women (Figure 2A), cycling speed remained stable at $23.6(s=2.5)$ $\mathrm{km} \cdot \mathrm{h}^{-1}(P>0.05)$ across years (Table 2). For the annual three fastest women (Figure 2B), cycling speed increased from $11.0(s=0.3) \mathrm{km} \cdot \mathrm{h}^{-1}(1984)$ to $24.2(s=0.2) \mathrm{km} \cdot \mathrm{h}^{-1}$ (Table 2). In the annual fastest men (Figure 2C), cycling speed increased from $14.9 \mathrm{~km} \cdot \mathrm{h}^{-1}$ to $27.7 \mathrm{~km} \cdot \mathrm{h}^{-1}$ (Table 2). For the annual three fastest men (Figure 2D), cycling speed increased from 14.6 $(s=0.3) \mathrm{km} \cdot \mathrm{h}^{-1}$ to $27.1(s=0.7) \mathrm{km} \cdot \mathrm{h}^{-1}$ (Table 2$)$. The changes in cycling speeds were nonlinear in the annual fastest women (i.e. polynomial regression $3^{\text {rd }}$ degree), in the annual fastest men (i.e. polynomial regression $5^{\text {th }}$ degree), in the annual three fastest men (i.e. polynomial regression $4^{\text {th }}$ degree) and in the annual three fastest women (i.e. polynomial regression $5^{\text {th }}$ degree) (Table 3).

\section{Changes in gender differences over time}

The gender difference in performance for the annual fastest cyclists (Figure 3A) remained unchanged at $14.3(s=8.2) \%(P>0.05)$ (Table 4). For the annual three fastest finishers (Figure 3B), the gender difference in performance decreased linearly from $26.2(s=0.5) \%$ (1984) to $10.7(s=1.9) \%(2012)$ (Table 5). 


\section{The fastest finishers ever}

Figure 4 presents the cycling speeds of the fastest women and men ever, the top three women and men ever and the top ten women and men ever. The fastest woman ever achieved a cycling speed of $28.41 \mathrm{~km} \cdot \mathrm{h}^{-1}$ and the fastest man ever of $30.0 \mathrm{~km} \cdot \mathrm{h}^{-1}$ with a gender difference of $5.3 \%$. For the top three ever cyclist, the cycling speed was $27.7(s=0.6) \mathrm{km} \cdot \mathrm{h}^{-1}$ for women and $29.9(s=0.1) \mathrm{km} \cdot \mathrm{h}^{-1}$ for men with a gender difference of $7.2(s=1.7) \%$. The top ten ever women achieved a cycling speed of $26.0(s=1.4) \mathrm{km} \cdot \mathrm{h}^{-1}$ and the top ten ever men $29.3(s=0.5) \mathrm{km} \cdot \mathrm{h}^{-1}$ with a gender difference of $11.3(s=3.1) \%$.

\section{Age of the person who produced the best performance in any given year}

The age of peak performance of the annual fastest finishers increased from 27 years to 53 years for women and from 30 years to 44 years for men (Figure 5) (Table 6). For the annual three fastest finishers (Figure 5B), the age of peak performance increased from $26(s=2)$ years (1984) to $43(s=11)$ years in women and from $33(s=6)$ years to $50(s=5)$ years in men (Table $6)$.

\section{The age of fastest finishers ever}

Figure 6 presents the age of the fastest women and men ever, the top three women and men ever and the top ten women and men ever. The fastest woman ever was 27 years old, the fastest man ever 41 years with a difference of $34.1 \%$. The top three women ever were 38.0 $(s=10.5)$ years, the fastest three men ever $36.3(s=4.5)$ years with a difference of $30.8(s=21.0)$ $\%$. Regarding the top ten finishers ever, women were $38.7(s=7.8)$ years old and men 35.9 $(s=9.6)$ years with a difference of $41.2(s=21.2) \%$. For both the top three and the top ten female and male finishers, the age of peak performance was not different between women and men. 


\section{Discussion}

\section{Women reduced the gender gap in ultra-cycling speed}

It has been reported that the gender difference in sports performance is non-linear (Reinboud 2004). However, in the present ultra-cyclists, the change in gender difference in performance was linear. The linear change in gender difference in the last 30 years may suggest that the fastest women will be able to narrow the gender gap in cycling speed in the 'Furnace Creek $508^{\prime}$ in the future.

The gender difference in cycling speed might be influenced by the low number of women where women accounted only for $11.2 \%$ of the participants and $10.1 \%$ of the finishers. Coast, Blevins, and Wilson (2004) compared the world's best running performances at distances from $100 \mathrm{~m}$ to $200 \mathrm{~km}$. Men were $12.4 \%$ faster than women and longer distances were associated with greater gender differences. The authors assumed that the results may be confounded by the reduced number of women in longer distance events (Coast, Blevins, \& Wilson, 2004).

However, the absolute values and the change in gender differences in performance are in line with previous results reported for cycling split times in triathlon (Lepers, 2008; Lepers, \& Maffiuletti, 2011) and duathlon (Rüst, et al., 2013a) races. Unfortunately, only a few studies investigated the change in gender difference in cycling performance across years. Lepers (2008) reported for the top ten finishers in 'Ironman Hawaii' between 1988 and 2007 an unchanged gender difference of $12.7(s=2.0) \%$ in cycling. In the present ultra-cyclists, the gender difference in cycling speed for the annual top three women and men with $10.7(s=1.9)$ 
$\%$ in 2012 was comparable to the gender difference in the $180 \mathrm{~km}$ cycling performance in 'Ironman Hawaii' with 12.7 (s=2.0) \% between 1988 and 2007 (Lepers, 2008) and the 17 $(s=3) \%$ in the $150 \mathrm{~km}$ cycling split in 'Powerman Zofingen' duathlon (Rüst, et al., 2013a) between 2002 and 2011.

The gender difference in endurance performance appears to be biological in origin (Cheuvront, Carter, Deruisseau, \& Moffatt, 2005). In distance running, success is largely determined by aerobic power and muscular strength. The gender gap in endurance performances is unlikely to narrow naturally since men have a larger aerobic power (Billat, Demarle, Slawinski, Paiva, \& Koralsztein, 2001) and a greater muscular strength (Gursoy, 2010). In several studies, the gender difference in performance amounted to $\sim 11-12 \%$ (Hunter, Stevens, Magennis, Skelton, \& Fauth, 2011; Schumacher, Mueller, \& Keul, 2001; Seiler, De Koning, \& Foster, 2007). In marathon running, men were 11.6 ( $s=1.8) \%$ faster than women when the world's fastest marathon times between 1983 and 2009 were analysed (Hunter, Stevens, Magennis, Skelton, \& Fauth, 2011). In the World Track Cycling Championships from 1979 to 1999 , the gender difference in performance was $11(s=1.8) \%$ for all disciplines and ages (Schumacher, Mueller, \& Keul, 2001). Sparling, O’Donnell, and Snow (1998) analysed the annual world best times from 1980 to 1996 for 1,500 m running and marathon. In 1,500m, the gender difference of $11.1(s=1.1) \%$ in the world best times was consistent from 1980 to 1996. In the marathon, the gender difference in the world best times of $11.2(s=0.9) \%$ was the same as for $1,500 \mathrm{~m}$. For swimming, the gender difference in performance for 1,500m freestyle was $11(s=1) \%$ (Tanaka, \& Seals, 1997) and $12.5(s=9.6)$ $\%$ in the 'English Channel Swim' (Fischer, Knechtle, Rüst, \& Rosemann, 2013). 
The gender difference of $14.3(s=8.2) \%$ for the annual fastest cyclists and $10.7(s=1.9) \%$ for the annual three fastest cyclists in 2012 was in line to findings in other ultra-endurance performances such as ultra-swimming (Eichenberger, et al., 2012a, 2013; Fischer, Knechtle, Rüst, \& Rosemann, 2013) and ultra-running (Hoffman, \& Wegelin, 2009). In the 'English Channel Swim', the gender difference for the annual three fastest swimmers was $12.5(s=9.6)$ \% between 1975 and 2011 (Fischer, Knechtle, Rüst, \& Rosemann, 2013). In the 'Lake Zurich Swim', the gender difference in swimming time was $11.5(s=11.6) \%$ for winners during 1987-2011 (Eichenberger, et al., 2013).

Recent studies showed that women were able to reduce the gender difference in swimming (Eichenberger, et al., 2012a, 2013; Fischer, Knechtle, Rüst, \& Rosemann, 2013; Rüst, Knechtle, Rosemann, \& Lepers, 2014a; Rüst, Lepers, Rosemann, \& Knechtle, 2014b; Vogt, Rüst, Rosemann, Lepers, \& Knechtle, 2013; Zingg, Rüst, Rosemann, Lepers, \& Knechtle, 2014), cycling (Gloor, Knechtle, Knechtle, Rüst, Haupt, Rosemann, \& Lepers, 2013; Shoak, Knechtle, Knechtle, Rüst, Rosemann, \& Lepers, 2013; Zingg, Knechtle, Rüst, Rosemann, \& Lepers, 2013a), running (Hoffman, \& Wegelin, 2009; Peter, Rüst, Knechtle, Rosemann, \& Lepers, 2014; Zingg, Knechtle, Rüst, Rosemann, \& Lepers, 2013a), and triathlon (Knechtle, Knechtle, \& Lepers, 2011a; Rüst, Knechtle, Rosemann, \& Lepers, 2012d) in recent years and confirmed the present trend that women were also able to reduce the gender gap in ultracycling.

The gender difference in ultra-performance might be explained by anthropometric differences such as differences in skeletal muscle mass and body fat between women and men. Male ultra-endurance athletes had a higher skeletal muscle mass than female ultra-endurance athletes (Knechtle, Baumann, Wirth, Knechtle, \& Rosemann, 2010a; Knechtle, et al., 2010b, 
2010c; 2010d; 2011b; Weitkunat, Knechtle, Knechtle, Rüst, \& Rosemann, 2012). Knechtle, Knechtle and Lepers (2011a) argued that the increase in gender difference with increasing length in ultra-performance such as an ultra-triathlon was most probably due to the lower skeletal muscle mass in women.

Another anthropometric variable for a successful endurance performance is percent body fat. Several studies investigated the correlation between percent body fat and ultra-performance (Knechtle, Knechtle, Lepers, \& Rosemann, 2010g; Knechtle, Knechtle, Rosemann, \& Lepers, 2010e, Knechtle, Wirth, Rüst, \& Rosemann, 2011d; Schmid, et al., 2012). Lower body fat was associated with faster race times in men (Knechtle, Knechtle, Rüst, \& Rosemann, 2011c; Rüst, Knechtle, Knechtle, \& Rosemann, 2012a). The average body fat percentage for female and male $100 \mathrm{~km}$ ultra-marathoners was $\sim 26.8 \%(20.0-31.4 \%)$ or $\sim 17.0 \mathrm{~kg}$ and $\sim 16.1 \%$ or $\sim 11.9 \mathrm{~kg}$, respectively (Knechtle, Knechtle, Rosemann, \& Lepers, 2010f; Knechtle, Knechtle, Lepers, \& Rosemann, 2010g). The higher skeletal muscle mass and the lower percent body fat in men may support the theory of a biological based performance difference of female and male ultra-endurance athletes.

Another explanation for the gender difference in participation and performance in ultraendurance could be a difference in motivation between men and women to compete in ultraendurance. Personality, motivation, and goal orientation have been investigated in endurance athletes such as runners (Bond, 2005) and participants in different disciplines (Frederick, \& Ryan, 1993; Gill, \& Overdorf, 1996). Women were motivated to exercise regularly to reduce body fat, to increase physical fitness, or to improve social interactions (Bond, 2005; Frederick, \& Ryan, 1993; Gill, \& Overdorf, 1996; Hodge, Allen, \& Smellie, 2008; Levy, 2002). The aspect of competing and winning seemed to be of lower importance for women 
compared to men. For example, for female marathoners, the aspect of social affiliation and improving physical fitness was more important than athletic achievement and personal accomplishment (Masters, \& Ogles, 1995; Ogles, \& Masters, 2003). Krouse, Ransdell, Lucas, and Pritchard (2011) investigated the influence of motivation, goal orientation and training in female ultra-marathoners. General health orientation and psychological coping were the strongest motivational factors for female ultra-marathoners.

For men, the motivation to compete in sport might be different compared to women. According to Lombardo (2012) the most popular modern male sports require skills needed for success in male-to-male physical competition, primitive hunting and warfare. Male champion athletes obtain a high status and reproductive opportunities in ways that parallel those gained by successful primitive hunters and warriors. Men pay closer attention than do women to male sports to evaluate potential allies and rivals. Male sports became culturally more important when opportunities to evaluate potential allies and rivals declined as both the survival importance of hunting and the proportion of men who experience combat decreased. The characteristics of primitive and modern sports are more consistent with these predictions than those generated by intersexual sexual selection theories of sport (Lombardo, 2012).

In this study, we included ambient air temperatures as co-variables to investigate a potential influence of environmental temperatures on performance since it has been reported for runners (Parise, \& Hoffman, 2011; Wegelin, \& Hoffman, 2011) and cyclists (Abbiss, et al., 2011; Pfeiffer, \& Abbiss, 2011) that ambient air temperatures have an influence on performance. Environmental temperatures may also influence cycling (Pfeiffer, \& Abbiss, 2011) and swimming (Eichenberger, et al., 2013) performance. Cycling performance was impaired at air temperatures of $>30^{\circ} \mathrm{C}$ in a $20 \mathrm{~km}$ time trial (Pfeiffer, \& Abbiss, 2011). For swimmers in a $26.4 \mathrm{~km}$ open-water ultra-swim competing at water temperatures of 16.2-25.9 
${ }^{\circ} \mathrm{C}$, water temperature was significantly and negatively associated with performance in the fastest swimmers (Eichenberger, et al., 2013). However, in the present ultra-cyclists, both performances and sex differences in performance were not influenced by environmental temperatures. Death Valley is famous as the hottest place on earth and driest place in North America (www.nps.gov/deva/naturescience/weather-and-climate.htm). The world record highest air temperature of $57{ }^{\circ} \mathrm{C}$ was recorded at Furnace Creek on July 10, 1913. Summer temperatures often top $49{ }^{\circ} \mathrm{C}$ in the shade with overnight lows dipping into the mid-30s ${ }^{\circ} \mathrm{C}$. (www.nps.gov/deva/naturescience/weather-and-climate.htm). The kind of exercise and the duration might be of importance whether performance will be impaired or not by ambient temperatures. In marathon running at temperatures from $5{ }^{\circ} \mathrm{C}$ to $25^{\circ} \mathrm{C}$, race times became slower for women and men with increasing temperature with no obvious differences between the genders (Ely, Cheuvront, Roberts, \& Montain, 2007). In a 164km road cycling event held during summer where the ambient temperature was $>39.0^{\circ} \mathrm{C}$ during the final two hours mean finish times for men and women were similar (Armstrong, et al., 2012). The aspect of previous experience in ultra-endurance needs also to be considered. Successful ultraendurance athletes such as ultra-marathoners (Hoffman \& Krishnan, 2013; Knechtle, Knechtle, Rosemann, \& Lepers, 2011f), ultra-triathletes (Herbst, et al., 2011), and ultramountain bikers (Knechtle, Knechtle, Rosemann, \& Senn, 2011g) have a large previous experience before they successfully compete in ultra-endurance races.

Changes in cycling speeds were non-linearly in these athletes. Polynomials of $5^{\text {th }}$ order have no biological or physiological meaning. However, a non-linear increase in cycling speed suggests that these athletes have reached their limits in this race and further improvements are rather unlikely. Future research might consider fitting more appropriate asymptotic models 
similar to those used by Nevill and Whyte (2005) when modelling world record running speeds.

\section{Winning performers become older and faster across years}

The second aim was to identify the age of peak ultra-cycling speed in a non-drafting ultracycling race. The age of the annual fastest finishers increased across years for women and men and the fastest cycling speeds were attained at the age of $\sim 30-40$ years for women and men. Generally, the age of peak performance in endurance athletes is not different between the genders for different disciplines such as running (Eichenberger, Knechtle, Rüst, Rosemann, \& Lepers, 2012b; Hunter, Stevens, Magennis, Skelton, \& Fauth, 2011), and triathlon (Gallmann, Knechtle, Rüst, Rosemann, \& Lepers, 2014; Knechtle, Rüst, Rosemann, \& Lepers, 2012c; Meili, Knechtle, Rüst, Rosemann, \& Lepers, 2013; Rüst, Knechtle, Knechtle, Rosemann, \& Lepers, 2012c).

The age of the best performance between 30-40 years for both women and men is a common finding in ultra-endurance athletes such as triathletes (Meili, Knechtle, Rüst, Rosemann, \& Lepers, 2013; Stiefel, Knechtle, Rüst, Rosemann, \& Lepers, 2013), runners (Eichenberger, Knechtle, Rüst, Rosemann, \& Lepers, 2012b; Zingg, Rüst, Lepers, Rosemann, \& Knechtle, 2013b) and cyclists (Zingg, Knechtle, Rüst, Rosemann, \& Lepers, 2013a). The top cyclists in the 'Furnace Creek 508' where therefore older than elite marathoners (Hunter, Stevens, Magennis, Skelton, \& Fauth, 2011) but at a similar age as reported for mountain ultramarathoners (Eichenberger, Knechtle, Rüst, Rosemann, \& Lepers, 2012b) and ultra-triathletes (Knechtle, Rüst, Knechtle, Rosemann, \& Lepers, 2012b). 
An increase in the age of the best performance across years has also been reported for ultraendurance athletes such as triathletes (Gallmann, Knechtle, Rüst, Rosemann, \& Lepers, 2014; Knechtle, Rüst, Rosemann, \& Lepers, 2012c; Meili, Knechtle, Rüst, Rosemann, \& Lepers, 2013; Rüst, Knechtle, Knechtle, Rosemann, \& Lepers, 2012c) and mountain ultramarathoners (Eichenberger, Knechtle, Rüst, Rosemann, \& Lepers, 2012b). Especially for triathletes, it has been shown that master triathletes showed relative improvements in their performances across the three triathlon disciplines and overall race times over the past three decades. This raises the question whether older male and female triathletes have yet reached their performance limits (Lepers, Knechtle, \& Stapley, 2013).

Age (Knechtle, Knechtle, Lepers, \& Rosemann, 2010g) and previous experience (i.e. number of years competing as an elite athlete, fast personal best time in races of shorter length or races of the same length, number of completed races of shorter or the same length) (Knechtle, Knechtle, Rüst, Rosemann, \&Lepers, 2011e; Rüst, Knechtle, Knechtle, \& Rosemann, 2012a) seemed to play a major role in succeeding in ultra-endurance performance and could therefore be a crucial factor in a $818 \mathrm{~km}$ cycling event such as the 'Furnace Creek 508'. In ultramarathoners, the number of previously completed marathons was higher than the number of completed marathons in marathoners and successful ultra-marathoners have $7.6(\mathrm{~s}=6.3)$ years of experience in ultra-running (Knechtle, 2012). Shaw and Ostrow (2005) investigated motivational factors (i.e. intrinsic motivation, such as enjoyment, perceptions of ability, commitment, etc. and extrinsic motivation) especially in older, i.e. >35 years, athletes and found them to be critical factors in accomplishing ultra-endurance performances. Hodge, Allen, and Smellie (2008) explored different social factor in athletes aged between 28 and 77 years. They found especially in athletes $>35$ years that intrinsic motivation was absolutely necessary to compete for $20 \mathrm{~h}$ plus without getting public attention. Master athletes enjoyed 
their participation, they were committed, they had high perceptions of ability and belonging, and they were predominantly intrinsically motivated.

\section{Conclusions}

To summarize, these results suggest that $(i)$ women will be able to narrow the gender gap in cycling speed in the 'Furnace Creek 508' race in the future due to the linear decrease in gender difference and (ii) the maturity of these athletes has changed during the last three decades with the fastest finishers becoming older across the years. The non-linear increase in cycling speeds suggests, however, that these athletes have reached their limits in the 'Furnace Creek 508' and further improvements are rather unlikely. Future studies may compare anthropometric and physiological characteristics of female and male ultra-cyclists. 


\section{References}

Abbiss, C.R., Burnett, A., Nosaka, K., Green, J.P., Foster, J.K., \& Laursen, PB. (2010). Effect of hot versus cold climates on power output, muscle activation, and perceived fatigue during a dynamic 100-km cycling trial. Journal of Sports Sciences, 28, 117-125.

Armstrong, L.E., Casa, D.J., Emmanuel, H., Ganio, M.S., Klau, J.F., Lee, E.C., Maresh, C.M., McDermott, B.P., Stearns, R.L., Vingren, J.L., Wingo, J.E., Williamson, K.H., \& Yamamoto, L.M (2012). Nutritional, physiological, and perceptual responses during a summer ultraendurance cycling event. Journal of Strength and Conditioning Research, 26, 307-318.

Bam, J., Noakes, T.D., Juritz, J., \& Dennis, S.C. (1997). Could women outrun men in ultramarathon races? Medicine and Science in Sports and Exercise, 29, 244-247.

Billat, V.L., Demarle, A., Slawinski, J., Paiva, M., \& Koralsztein, J.P. (2011). Physical and training characteristics of top-class marathon runners. Medicine and Science in Sports and Exercise, 3, 2089-2097.

Bond, K. (2005). Running for their lives: A qualitative analysis of the exercise experience of female recreational runners. Women in Sport and Physical Activity Journal, 14, 69-82.

Cheuvront, S.N., Carter, R., Deruisseau, K.C., \& Moffatt, R.J. (2005). Running performance differences between men and women: an update. Sports Medicine, 35, 1017-1024.

Coast, J.R., Blevins, J.S., \& Wilson, B.A. (2004). Do gender differences in running performance disappear with distance? Canadian Journal of Applied Physiology, 29, 139-145.

Eichenberger, E., Knechtle, B., Knechtle, P., Rüst, C.A., Rosemann, T., \& Lepers, R. (2012a). Best performances by men and women open-water swimmers during the 'English Channel Swim' from 1900 to 2010. Journal of Sports Sciences, 30, 1295-1301.

Eichenberger, E., Knechtle, B., Rüst, C.A., Rosemann, T., \& Lepers, R. (2012b). Age and gender interactions in mountain ultra-marathon running - the 'Swiss Alpine Marathon'. Open Access Journal of Sports Medicine, 3, 73-80.

Eichenberger, E., Knechtle, B., Knechtle, P., Rüst, C.A., Rosemann, T., \& Lepers, R. (2012c). No gender difference in peak performance in ultra-endurance swimming performance Analysis of the 'Zurich 12-h Swim' from 1996 to 2010. Chinese Journal of Physiology, 55, 346-351

Eichenberger, E., Knechtle, B., Knechtle, P., Rüst, C.A., Rosemann, T., Lepers, R., \& Senn, O. (2013). Sex difference in open-water ultra-swim performance in the longest freshwater lake swim in Europe. Journal of Strength and Conditioning Research, 27, 1362-1369.

Ely, M.R., Cheuvront, S.N., Roberts, W.O., \& Montain, S.J. (2007). Impact of weather on marathon-running performance. Medicine and Science in Sports and Exercise, 39, 487-493. 
Fischer, G., Knechtle, B., Rüst, C.A., \& Rosemann, T. (2013). Male swimmers cross the 'English Channel' faster than females. Scandinavian Journal of Medicine and Science in Sports, 23, e48-55.

Frederick, C., \& Ryan, R. (1993). Differences in motivation for sport and exercise and their relations with participation and mental health. Journal of Sport Behavior, 16, 124-146.

Gallmann, D., Knechtle, B., Rüst, C.A., Rosemann, T., \& Lepers, R. (2014). Elite triathletes in 'Ironman Hawaii' get older but faster. Age (Dordr), 36, 407-416.

Gill, K., \& Overdorf, V. (1994). Incentives for exercise in younger and older women. Journal of Sport Behavior, 17, 87-97.

Gloor, R.U., Knechtle, B., Knechtle, P., Rüst, C.A., Haupt, S., Rosemann, T., \& Lepers, R. (2013). Sex-related trends in participation and performance in the 'Swiss Bike Masters' from 1994-2012. Perceptual and Motor Skills, 116, 640-654.

Gursoy, R. (2010). Sex differences in relations of muscle power, lung function, and reaction time in athletes. Perceptual and Motor Skills, 110, 714-720.

Herbst, L., Knechtle, B., Lopez, C.L., Andonie, J.L., Fraire, O.S., Kohler, G., Rüst, C.A., \& Rosemann, T. (2011). Pacing strategy and change in body composition during a Deca Iron Triathlon. Chinese Journal of Physiology, 54, 255-263.

Hodge, K., Allen, J.B., \& Smellie, L. (2008). Motivation in masters sport: Achievement and social goals. Psychology of Sport and Exercise, 9, 157-176.

Hoffman, M.D. (2008). Ultramarathon trail running comparison of performance-matched men and women. Medicine and Science in Sports and Exercise, 40, 1681-1686.

Hoffman, M.D. (2010). Performance trends in 161-km ultramarathons. International Journal of Sports Medicine, 31, 31-37.

Hoffman, M.D., \& Krishnan, E. (2013). Exercise behavior of ultramarathon runners: baseline findings from the ULTRA study. Journal of Strength and Conditioning Research, 27, 29392945.

Hoffman, M.D., \& Wegelin, J.A. (2009). The Western States 100-Mile Endurance Run: participation and performance trends. Medicine and Science in Sports and Exercise, 41, 21912198.

Hunter, S.K., Stevens, A.A., Magennis, K., Skelton, K.W., \& Fauth, M. (2011). Is there a sex difference in the age of elite marathon runners? Medicine and Science in Sports and Exercise, $43,656-664$.

Knechtle, B. (2012). Ultramarathon runners: nature or nurture? International Journal of Sports Physiology and Performance, 7, 310-312. 
Knechtle, B., Enggist, A., \& Jehle, T. (2005). Energy turnover at the Race Across AMerica (RAAM) - a case report. International Journal of Sports Medicine, 26, 499-503.

Knechtle, B., Baumann, B., Wirth, A., Knechtle, P., \& Rosemann, T. (2010a). Male ironman triathletes lose skeletal muscle mass. Asia Pacific Journal of Clinical Nutrition, 19, 91-97.

Knechtle, B., Wirth, A., Baumann, B., Knechtle, P., Kohler, G., Rosemann, T., \& Senn, O. (2010b). An Ironman triathlon does not lead to a change in body mass in female triathletes. Research in Sports Medicine, 18, 115-126.

Knechtle, B., Senn, O., Imoberdorf, R., Joleska, I., Wirth, A., Knechtle, P., \& Rosemann, T. (2010c). Maintained total body water content and serum sodium concentrations despite body mass loss in female ultra-runners drinking ad libitum during a $100 \mathrm{~km}$ race. Asia Pacific Journal of Clinical Nutrition, 19, 83-90.

Knechtle, B., Wirth, A., Baumann, B., Knechtle, P., Rosemann, T., \& Senn, O. (2010d). Differential correlations between anthropometry, training volume, and performance in male and female Ironman triathletes. Journal of Strength and Conditioning Research, 24, 27852793.

Knechtle, B., Knechtle, P., Rosemann, T., \& Lepers, R. (2010e). Is body fat a predictor of race time in female long-distance inline skaters? Asian Journal of Sports Medicine, 1, 131136.

Knechtle, B., Knechtle, P., Rosemann, T., \& Lepers, R. (2010f). Predictor variables for a 100$\mathrm{km}$ race time in female ultra-marathoners. Medicina Sportiva, 14, 214-220.

Knechtle, B., Knechtle, P., Lepers, R., \& Rosemann, T. (2010g). Predictor variables for a 100-km race time in male ultra-marathoners. Perceptual and Motor Skills, 111, 681-693.

Knechtle, B., Knechtle, P., \& Lepers, R. (2011a). Participation and performance trends in ultra-triathlons from 1985 to 2009. Scandinavian Journal of Medicine and Science in Sports, 21, e82-e90.

Knechtle, B., Senn, O., Imoberdorf, R., Joleska, I., Wirth, A., Knechtle, P., \& Rosemann, T. (2011b). No fluid overload in male ultra-runners during a $100 \mathrm{~km}$ ultra-run. Research in Sports Medicine, 19, 14-27.

Knechtle, B., Knechtle, P., Rüst, C.A., \& Rosemann, T. (2011c). A comparison of anthropometric and training characteristics of Ironman triathletes and Triple Iron ultratriathletes. Journal of Sports Sciences, 29, 1373-1380.

Knechtle, B., Wirth, A., Rüst, C.A., \& Rosemann, T. (2011d). The relationship between anthropometry and split performance in recreational male Ironman triathletes. Asian Journal of Sports Medicine, 2, 23-30.

Knechtle, B., Knechtle, P., Rüst, C.A., Rosemann, T., \& Lepers, R. (2011e). Finishers and nonfinishers in the 'Swiss Cycling Marathon ' to qualify for the 'Race Across America '. Journal of Strength and Conditioning Research, 25, 3257-3263. 
Knechtle, B., Knechtle, P., Rosemann, T., \& Lepers, R. (2011f). Personal best marathon time and longest training run, not anthropometry, predict performance in recreational 24-hour ultrarunners. Journal of Strength and Conditioning Research, 25, 2212-2218.

Knechtle, B., Knechtle, P., Rosemann, T., \& Senn, O. (2011g). Personal best time and training volume, not anthropometry, is related to race performance in the 'Swiss Bike Masters' mountain bike ultramarathon. Journal of Strength and Conditioning Research, 25, 1312-1317.

Knechtle, B., Rosemann, T., Lepers, R., \& Rüst, C.A. (2014). Women outperform men in ultra-distance swimming - the 'Manhattan Island Marathon Swim' from 1983 to 2013. International Journal of Sports Physiology and Performance 2014 Feb 28. [Epub ahead of print]

Knechtle, B., Rüst, C.A., Knechtle, P., Rosemann, T. \& Lepers, R. (2012b). Age-related changes in ultra-triathlon performances. Extreme Physiology and Medicine, 1, 5.

Knechtle, B., Rüst, C.A., Rosemann, T., \& Lepers, R. (2012c).Age and gender differences in half-Ironman triathlon performances - the Ironman 70.3 Switzerland from 2007 to 2010. Open Access Journal of Sports Medicine, 3, 59-66.

Knechtle, B., Wirth, A., Knechtle, P., Rüst, C.A., Rosemann, T., \& Lepers, R. (2012a). No improvement in race performance by naps in male ultra-endurance cyclists in a $600-\mathrm{km}$ ultracycling race. Chinese Journal of Physiology, 55, 125-133.

Krouse, R.Z., Ransdell, L.B., Lucas, S.M., \& Pritchard, M.E. (2011). Motivation, goal orientation and training habits of women ultrarunners. Journal of Strength and Conditioning Research, 25, 2835-3842.

Lepers, R. (2008). Analysis of Hawaii ironman performances in elite triathletes from 1981 to 2007. Medicine and Science in Sports and Exercise, 40, 1828-1234.

Lepers, R., \& Maffiuletti, N.A. (2011). Age and gender interactions in ultraendurance performance: insight from the triathlon. Medicine and Science in Sports and Exercise, 43, 134-139.

Lepers, R., Knechtle, B., \& Stapley, P.J. (2013). Trends in triathlon performance: effects of sex and age. Sports Medicine, 43, 851-863.

Levy, S. (2002). Women and the personal meaning of competition: A qualitative investigation. Women in Sport and Physical Activity Journal, 11, 107-119.

Lombardo, M.P. (2012). On the evolution of sport. Evolutionary Psychology, 10, 1-28.

Masters, K., \& Ogles, B. (1995). An investigation of the different motivations of marathon runners with varying degrees of experience. Journal of Sport Behavior, 18, 69-79.

Meili, D., Knechtle, B., Rüst, C.A., Rosemann, T., \& Lepers, R. (2013). Participation and performance trends in 'Ultraman Hawaii' from 1983 to 2012. Extreme Physiology and Medicine, 2, 25. 
Nevill, A.M., \& Whyte, G. (2005). Are there limits to running world-records? Medicine and Science in Sports and Exercise, 37, 1785-1788.

Ogles, B., \& Masters, K. (2003). A typology of marathon runners based on cluster analysis of motivation. Journal of Sport Behavior, 26, 69-85.

Parise, C.A., \& Hoffman, M.D. (2011). Influence of temperature and performance level on pacing a $161 \mathrm{~km}$ trail ultramarathon. International Journal of Sports Physiology and Performance, 6, 243-251.

Peiffer, J.J., \& Abbiss, C.R. (2011). Influence of environmental temperature on $40 \mathrm{~km}$ cycling time-trial performance. International Journal of Sports Physiology and Performance, 6, 208220.

Peter, L., Rüst, C.A., Knechtle, B., Rosemann, T., \& Lepers, R. (2014). Sex differences in 24hour ultra-marathon performance - A retrospective data analysis from 1977 to 2012. Clinics (Sao Paulo), 69, 38-46.

Pozzi, L., Knechtle, B., Knechtle, P., Rosemann, T., Lepers, R., \& Rüst, C.A. (2014). Sex and age-related differences in performance in a 24-hour ultra-cycling draft-legal event - a crosssectional data analysis. BMC Sports Science, Medicine and Rehabilitation, 6,19.

Reinboud, W. (2004). Linear models can't keep up with sport gender gap. Nature, 432(7014), 147.

Rüst, C. A., Knechtle, B., Knechtle, P., \& Rosemann, T. (2012a). Similarities and differences in anthropometry and training between recreational male $100-\mathrm{km}$ ultra-marathoners and marathoners. Journal of Sports Sciences, 30, 1249-1257.

Rüst, C. A., Knechtle, B., Knechtle, P., Rosemann, T., \& Lepers, R. (2012b). Participation and performance trends in Triple Iron ultra-triathlon - a cross-sectional and longitudinal data analysis. Asian Journal of Sports Medicine, 3, 145-152.

Rüst, C. A., Knechtle, B., Knechtle, P., Rosemann, T., \& Lepers, R. (2012c). Age of peak performance in elite male and female Ironman triathletes competing in Ironman Switzerland, a qualifier for the Ironman world championship, Ironman Hawaii, from 1995 to 2011. Open Access Journal of Sports Medicine, 3, 175-182.

Rüst, C. A., Knechtle, B., Rosemann, T., \& Lepers, R. (2012d). Sex difference in race performance and age of peak performance in the Ironman Triathlon World Championship from 1983 to 2012. Extreme Physiology and Medicine, 1, 15.

Rüst, C. A., Knechtle, B., Knechtle, P., Pfeifer, S., Rosemann, T., Lepers, R., \& Senn, O. (2013a). Gender difference and age-related changes in performance at the long distance duathlon World Championships. Journal of Strength and Conditioning Research, 27, 293301. 
Rüst, C. A., Knechtle, B., Rosemann, T., \& Lepers, R. (2013b). Men cross America faster than women--the 'Race Across America' from 1982 to 2012. International Journal of Sports Physiology and Performance, 8, 611-7.

Rüst, C. A., Knechtle, B., Rosemann, T., \& Lepers, R. (2014a). Women reduced the sex difference in open-water ultra-distance swimming La Traversée Internationale du Lac St-Jean, 1955-2012. Applied Physiology, Nutrition, and Metabolism, 39, 270-273.

Rüst, C. A., Lepers, R., Rosemann, T., \& Knechtle, B. (2014b). Will women soon outperform men in open-water ultra-distance swimming in the 'Maratona del Golfo Capri-Napoli'? Springerplus, 3, 86.

Schmid, W., Knechtle, B., Knechtle, P., Barandun, U., Rüst, C.A., Rosemann, T., \& Lepers, R. (2012). Predictor variables for marathon race time in recreational female runners. Asian Journal of Sports Medicine, 2, 90-98.

Schulz, R., \& Curnow, C. (1988). Peak performance and age among superathletes: track and field, swimming, baseball, tennis, and golf. Journal of Gerontology, 43, 113-120.

Schumacher, Y.O., Mueller, P., \& Keul, J. (2001). Development of peak performance in track cycling. The Journal of Sports Medicine and Physical Fitness, 41, 139-146.

Seiler, S., De Koning, J.J., \& Foster, C. (2007). The fall and rise of the gender difference in elite anaerobic performance 1952-2006. Medicine and Science in Sports and Exercise, 39, 534-540.

Shaw, K.L., \& Ostrow, A. (2005). Motivation and psychological skills in the senior athlete. European Review of Aging and Physical Activity, 2, 22-34.

Shoak, M.A., Knechtle, B., Knechtle, P., Rüst, C.A., Rosemann, T., \& Lepers, R. (2013). Participation and performance trends in ultracycling. Open Access Journal of Sports Medicine, 4, 41-51.

Sparling, P.B., O'Donnell, E.M., \& Snow, T.K. (1998). The gender difference in distance running performance has plateaued: an analysis of world rankings from 1980 to 1996. Medicine and Science in Sports and Exercise, 30, 1725-1729.

Speechly, D.P., Taylor, S.R., \& Rogers, G.G. (1996). Differences in ultraendurance exercise in performance-matched male and female runners. Medicine and Science in Sports and Exercise, 28, 359-365.

Stiefel, M., Knechtle, B., Rüst, C.A., Rosemann, T., \& Lepers, R. (2013). The age of peak performance in Ironman triathlon: a cross-sectional and longitudinal data analysis. Extreme Physiology and Medicine, 2, 27.

Tanaka, H., \& Seals, D.R. (1997). Age and gender interactions in physiological functional capacity: insight from swimming performance. Journal of Applied Physiology, 82, 846-851. 
Vogt, P., Rüst, C.A., Rosemann, T., Lepers, R., \& Knechtle, B. (2013). Analysis of 10 km swimming performance of elite male and female open-water swimmers. Springerplus, 2, 603.

Wegelin, J.A., \& Hoffman, M.D. (2011). Variables associated with odds of finishing and finish time in a 161-km ultramarathon. European Journal of Applied Physiology, 111, 145153.

Weitkunat, T., Knechtle, B., Knechtle, P., Rüst, C.A., \& Rosemann, T. (2012). Body composition and hydration status changes in male and female open-water swimmers during an ultra-endurance event. Journal of Sports Sciences, 30, 1003-1013.

Zingg, M., Knechtle, B., Rüst, C.A., Rosemann, T., Lepers, R. (2013a). Age and gender difference in non-drafting ultra-endurance cycling performance - the 'Swiss Cycling Marathon'. Extreme Physiology and Medicine, 2, 18.

Zingg, M., Rüst, C.A., Lepers, R., Rosemann, T., \& Knechtle, B. (2013b). Master runners dominate 24-h ultramarathons worldwide-a retrospective data analysis from 1998 to 2011. Extreme Physiology and Medicine, 2, 21.

Zingg, M.A., Rüst, C.A., Rosemann, T., Lepers, R., \& Knechtle, B. (2014). Analysis of swimming performance in FINA World Cup long-distance open water races. Extreme Physiology and Medicine, 3, 2.

Zaryski, C., \& Smith, D.J. (2005). Training principles and issues for ultra-endurance athletes. Current Sports Medicine Reports, 4, 165-170. 


\begin{tabular}{|c|c|c|c|}
\hline & $\begin{array}{c}\text { Highest } \\
\text { temperatures } \\
\left({ }^{\circ} \mathrm{C}\right) \\
\end{array}$ & $\begin{array}{c}\text { Median } \\
\text { temperatures } \\
\left({ }^{\circ} \mathrm{C}\right) \\
\end{array}$ & $\begin{array}{c}\text { Lowest } \\
\text { temperatures } \\
\left({ }^{\circ} \mathrm{C}\right) \\
\end{array}$ \\
\hline 1983 & 35 & 27 & 20 \\
\hline 1984 & 39 & 31 & 24 \\
\hline 1985 & 35 & 27 & 20 \\
\hline 1986 & 35 & 28 & 21 \\
\hline 1987 & 34 & 27 & 22 \\
\hline 1988 & 35 & 28 & 21 \\
\hline 1989 & 28 & 23 & 19 \\
\hline 1990 & 36 & 27 & 17 \\
\hline 1991 & 29 & 21 & 14 \\
\hline 1992 & 31 & 22 & 14 \\
\hline 1993 & 40 & 32 & 23 \\
\hline 1994 & 32 & 26 & 18 \\
\hline 1995 & 28 & 23 & 19 \\
\hline 1996 & 27 & 22 & 18 \\
\hline 1997 & 36 & 29 & 23 \\
\hline 1998 & 33 & 26 & 18 \\
\hline 1999 & 39 & 31 & 23 \\
\hline 2000 & 32 & 24 & 17 \\
\hline 2001 & 37 & 27 & 17 \\
\hline 2002 & 37 & 29 & 21 \\
\hline 2003 & 38 & 31 & 24 \\
\hline 2004 & 32 & 24 & 17 \\
\hline 2005 & 33 & 24 & 16 \\
\hline 2006 & 32 & 25 & 18 \\
\hline 2007 & 28 & 23 & 16 \\
\hline 2008 & 32 & 24 & 16 \\
\hline 2009 & 27 & 21 & 15 \\
\hline 2010 & 34 & 28 & 22 \\
\hline 2011 & 31 & 23 & 16 \\
\hline 2012 & 36 & 28 & 19 \\
\hline
\end{tabular}

Table 1: The highest, the median and the lowest temperature during the races. Historical weather data were retrieved from www.almanac.com/weather/history 


\begin{tabular}{|c|c|c|c|c|c|}
\hline Model & $\beta$ & SE $(\beta)$ & Stand. $\beta$ & $\mathbf{T}$ & $p$ \\
\hline \multicolumn{6}{|c|}{ Annual fastest women } \\
\hline 1 & 0.245 & 0.073 & 0.535 & 3.352 & 0.002 \\
\hline 2 & 0.245 & 0.073 & 0.535 & 3.352 & 0.002 \\
\hline 3 & 0.153 & 0.104 & 0.334 & 1.474 & 0.152 \\
\hline 4 & 0.141 & 0.105 & 0.307 & 1.338 & 0.193 \\
\hline 5 & 0.136 & 0.101 & 0.297 & 1.346 & 0.190 \\
\hline 6 & 0.133 & 0.100 & 0.291 & 1.337 & 0.193 \\
\hline \multicolumn{6}{|c|}{ Annual three fastest women } \\
\hline 1 & 0.293 & 0.042 & 0.731 & 7.015 & $<0.001$ \\
\hline 2 & 0.293 & 0.042 & 0.731 & 7.015 & $<0.001$ \\
\hline 3 & 0.302 & 0.053 & 0.731 & 5.670 & $<0.001$ \\
\hline 4 & 0.266 & 0.059 & 0.644 & 4.493 & $<0.001$ \\
\hline 5 & 0.285 & 0.058 & 0.691 & 4.910 & $<0.001$ \\
\hline 6 & 0.300 & 0.058 & 0.727 & 5.157 & $<0.001$ \\
\hline \multicolumn{6}{|c|}{ Annual fastest men } \\
\hline $\mathbf{1}$ & 0.259 & 0.063 & 0.614 & 4.113 & $<0.001$ \\
\hline 2 & 0.259 & 0.063 & 0.614 & 4.113 & $<0.001$ \\
\hline 3 & 0.275 & 0.072 & 0.653 & 3.833 & $<0.001$ \\
\hline 4 & 0.246 & 0.077 & 0.583 & 3.203 & 0.004 \\
\hline 5 & 0.249 & 0.073 & 0.590 & 3.403 & 0.002 \\
\hline 6 & 0.250 & 0.071 & 0.593 & 3.521 & 0.002 \\
\hline \multicolumn{6}{|c|}{ Annual three fastest men } \\
\hline 1 & 0.239 & 0.036 & 0.575 & 6.598 & $<0.001$ \\
\hline 2 & 0.239 & 0.036 & 0.575 & 6.598 & $<0.001$ \\
\hline 3 & 0.225 & 0.040 & 0.543 & 5.661 & $<0.001$ \\
\hline 4 & 0.205 & 0.041 & 0.494 & 4.983 & $<0.001$ \\
\hline 5 & 0.206 & 0.040 & 0.498 & 5.209 & $<0.001$ \\
\hline 6 & 0.204 & 0.039 & 0.492 & 5.267 & $<0.001$ \\
\hline
\end{tabular}

Table 2: Multi-level regression analyses for changes in cycling speeds of the annual fastest and the annual three fastest female and male finishers (Model 1) with correction for multiple finishes (Model 2), the age of athletes with multiple finishes (Model 3), and with correction for the lowest (Model 4), the mean (Model 5), and the highest air temperatures (Model 6) during the race. 


\begin{tabular}{|c|c|c|c|c|c|c|c|c|c|}
\hline Cycling speed & $\begin{array}{l}\text { Kind of } \\
\text { regression }\end{array}$ & $\begin{array}{c}\text { Sum of } \\
\text { Squares }\end{array}$ & DOF & AICc & $\begin{array}{c}\text { Best } \\
\text { regression } \\
\text { AIC-Test }\end{array}$ & $\begin{array}{c}\text { Best } \\
\text { regression } \\
\text { F-Test }\end{array}$ & Delta & Probability & Likelihood \\
\hline \multirow[t]{3}{*}{ Annual fastest women } & polynomial & 152.4 & 26 & 55.6 & \multirow{3}{*}{ polynomial } & \multirow{3}{*}{ polynomial } & \multirow{3}{*}{18.9} & \multirow{3}{*}{$7.5 \mathrm{e}^{-05}$} & \multirow{3}{*}{$99.99 \%$} \\
\hline & & & & & & & & & \\
\hline & linear & 336.4 & 28 & 74.6 & & & & & \\
\hline \multirow[t]{2}{*}{ Annual three fastest women } & polynomial & 27.7 & 9 & 25.8 & \multirow[b]{2}{*}{ polynomial } & \multirow[b]{2}{*}{ polynomial } & \multirow[b]{2}{*}{2.7} & \multirow[b]{2}{*}{0.20} & \multirow[b]{2}{*}{$79.4 \%$} \\
\hline & linear & 86.4 & 13 & 28.5 & & & & & \\
\hline \multirow[t]{2}{*}{ Annual fastest men } & polynomial & 59.7 & 24 & 33.1 & \multirow[b]{2}{*}{ polynomial } & \multirow[b]{2}{*}{ polynomial } & \multirow[b]{2}{*}{32.5} & \multirow[b]{2}{*}{$8.6 \mathrm{e}^{-08}$} & \multirow[b]{2}{*}{$100 \%$} \\
\hline & linear & 249.3 & 28 & 65.6 & & & & & \\
\hline \multirow[t]{2}{*}{ Annual three fastest men } & polynomial & 92.8 & 25 & 43.5 & \multirow[b]{2}{*}{ polynomial } & \multirow[b]{2}{*}{ polynomial } & \multirow[b]{2}{*}{34.6} & \multirow[b]{2}{*}{$2.9 \mathrm{e}^{-08}$} & \multirow[b]{2}{*}{$100 \%$} \\
\hline & linear & 378.5 & 28 & 78.1 & & & & & \\
\hline
\end{tabular}

Table 3: Comparison of linear and non-linear regression analysis of changes in cycling speed across years to determine which model is the best 


\begin{tabular}{cccccc}
\hline Model & $\boldsymbol{\beta}$ & SE $(\boldsymbol{\beta})$ & Stand. $\boldsymbol{\beta}$ & $\mathbf{T}$ & $\boldsymbol{p}$ \\
\hline $\mathbf{1}$ & -0.146 & 0.167 & -0.163 & -0.873 & 0.390 \\
$\mathbf{2}$ & -0.146 & 0.167 & -0.163 & -0.873 & 0.390 \\
$\mathbf{3}$ & -0.125 & 0.178 & -0.139 & -0.700 & 0.490 \\
$\mathbf{4}$ & -0.111 & 0.171 & -0.124 & -0.648 & 0.522 \\
$\mathbf{5}$ & -0.118 & 0.171 & -0.131 & -0.687 & 0.498 \\
\hline $\mathbf{1}$ & -0.421 & 0.125 & -0.456 & -3.359 & 0.002 \\
$\mathbf{2}$ & -0.421 & 0.125 & -0.456 & -3.359 & 0.002 \\
$\mathbf{3}$ & -0.429 & 0.151 & -0.465 & -2.839 & 0.007 \\
$\mathbf{4}$ & -0.490 & 0.141 & -0.531 & -3.473 & 0.001 \\
$\mathbf{5}$ & -0.540 & 0.133 & -0.585 & -4.058 & $<0.001$ \\
\hline
\end{tabular}

Table 4: Multi-level regression analyses for changes in gender differences of the annual fastest and the annual three fastest finishers (Model 1) with correction for multiple finishes (Model 2), and with correction for the lowest (Model 3), the mean (Model 4), and the highest air temperatures (Model 5) during the race. 


\begin{tabular}{|c|c|c|c|c|c|c|c|c|c|}
\hline Gender difference & $\begin{array}{l}\text { Kind of } \\
\text { regression }\end{array}$ & $\begin{array}{c}\text { Sum of } \\
\text { Squares }\end{array}$ & DOF & AICC & $\begin{array}{c}\text { Best } \\
\text { regression } \\
\text { AIC-Test }\end{array}$ & $\begin{array}{c}\text { Best } \\
\text { regression } \\
\text { F-Test } \\
\end{array}$ & Delta & Probability & Likelihood \\
\hline \multirow{3}{*}{ Annual fastest } & polynomial & 1542.7 & 26 & 125.12 & \multirow{3}{*}{ linear } & \multirow{3}{*}{ linear } & \multirow{3}{*}{1.30} & \multirow{3}{*}{0.34} & \multirow{3}{*}{$65.72 \%$} \\
\hline & & & & & & & & & \\
\hline & linear & 1732.3 & 28 & 123.8 & & & & & \\
\hline \multirow[t]{3}{*}{ Annual three fastest } & polynomial & 498.4 & 8 & 75.0 & \multirow{3}{*}{ linear } & \multirow{3}{*}{ linear } & \multirow{3}{*}{20.0} & \multirow{3}{*}{$4.37 \mathrm{e}^{-05}$} & \multirow{3}{*}{$99.99 \%$} \\
\hline & & & & & & & & & \\
\hline & linear & 502.4 & 13 & 54.9 & & & & & \\
\hline
\end{tabular}

Table 5: Comparison of linear and non-linear regression analysis of changes in gender difference across years to determine which model is the best 


\begin{tabular}{|c|c|c|c|c|c|}
\hline Model & $\beta$ & SE $(\beta)$ & Stand. $\beta$ & $\mathbf{T}$ & $p$ \\
\hline \multicolumn{6}{|c|}{ Annual fastest men } \\
\hline $\mathbf{1}$ & 0.347 & 0.127 & 0.459 & 2.737 & 0.011 \\
\hline 2 & 0.347 & 0.127 & 0.459 & 2.737 & 0.011 \\
\hline \multicolumn{6}{|c|}{ Annual fastest women } \\
\hline 1 & 0.618 & 0.114 & 0.716 & 5.422 & $<0.001$ \\
\hline 2 & 0.618 & 0.114 & 0.716 & 5.422 & $<0.001$ \\
\hline \multicolumn{6}{|c|}{ Annual three fastest men } \\
\hline $\mathbf{1}$ & 0.368 & 0.086 & 0.415 & 4.273 & $<0.001$ \\
\hline 2 & 0.368 & 0.086 & 0.415 & 4.273 & $<0.001$ \\
\hline \multicolumn{6}{|c|}{ Annual three fastest women } \\
\hline 1 & 0.606 & 0.138 & 0.561 & 4.391 & $<0.001$ \\
\hline 2 & 0.606 & 0.138 & 0.561 & 4.391 & $<0.001$ \\
\hline
\end{tabular}

Table 6: Multi-level regression analyses for the changes in the age of the annual fastest and the annual three fastest female and male finishers across years (Model 1) and with correction for multiple finishes (Model 2) 


\section{Figure Captions}

Figure 1 Changes in the number of female, male, and overall finishers in the 'Furnace Creek 508' between 1983 and 2012

Figure 2 Changes in cycling speed of the annual fastest (Panel A) and the annual three fastest women (Panel B) and the annual fastest (Panel C) and annual three fastest men (Panel D) across years.

Figure 3 Changes in gender difference for the annual fastest (Panel A) and annual three fastest (Panel B) finishers across years.

Figure 4 Cycling speeds of the fastest women and men ever, the three fastest women and men ever, and the ten fastest women and men ever

Figure 5 Changes in the age of annual fastest female and male finishers (Panel A) and the annual three fastest female and male finishers (Panel B)

Figure 6 Age of the fastest women and men ever, the three fastest women and men ever, and the ten fastest women and men ever 


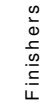

Figure 1 

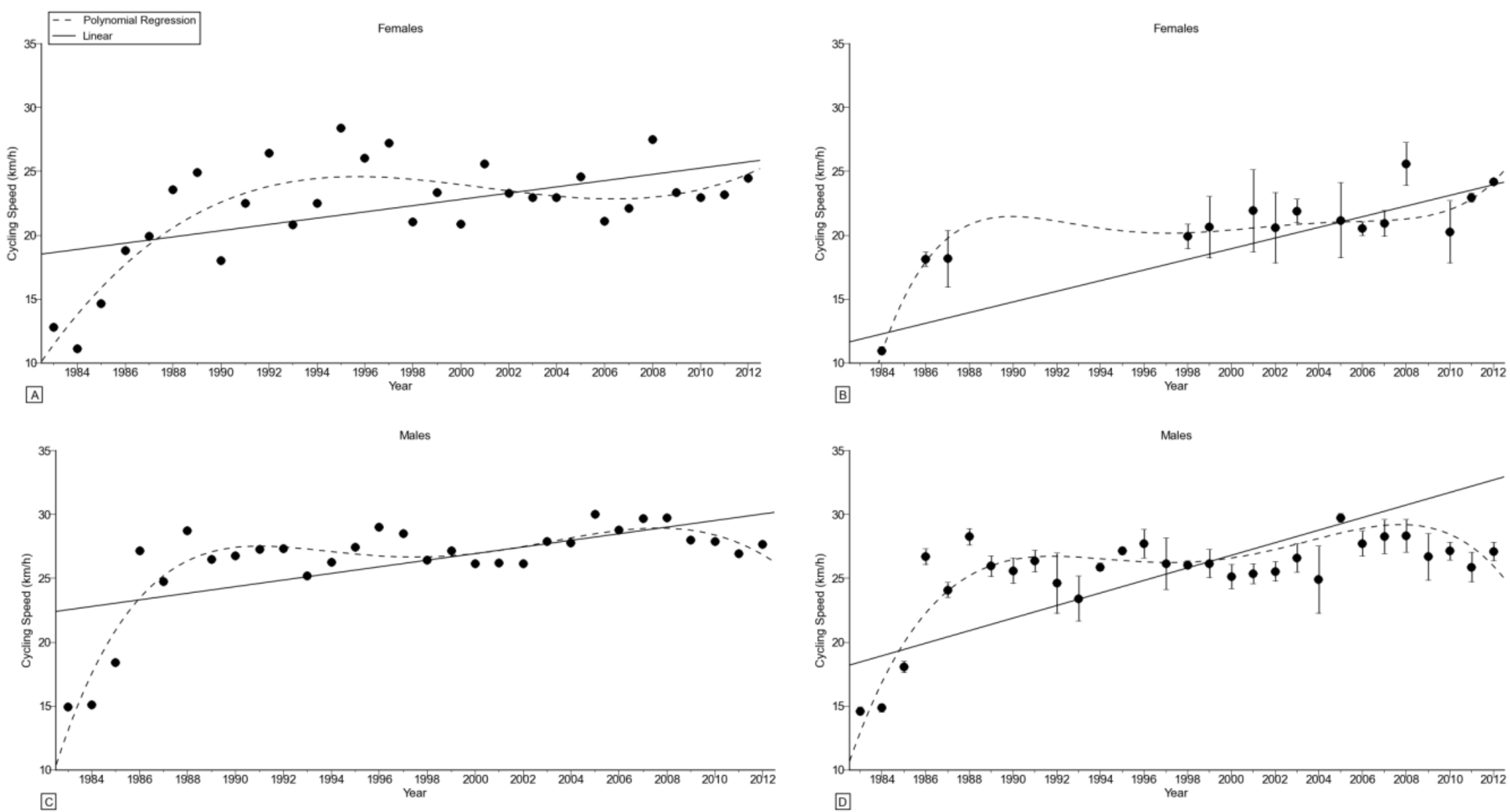

Figure 2 

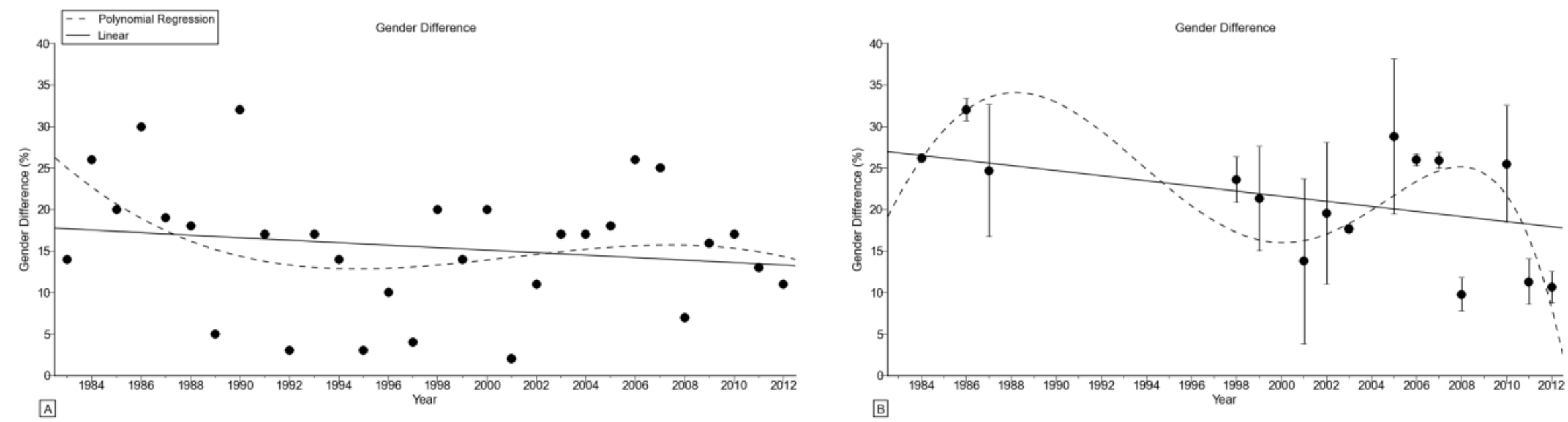

Figure 3 
0
0
0
0
0
$c$
0
0
0
0
0
0
0
0
0
0
0
0
0
0
0
0
0
0
0
0
0
0
0
0
0

Figure 4 
Figure 5 


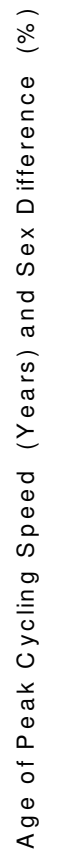

Figure 6 